\title{
Smart Materials Meet Multifunctional Biomedical Devices: Current and Prospective Implications for Nanomedicine
}

\author{
Giada Graziana Genchi ${ }^{1 *}$, Attilio Marino ${ }^{1}$, Christos Tapeinos ${ }^{1}$ and Gianni Ciofani, ${ }^{1,2 *}$ \\ ${ }^{1}$ Smart Bio-Interfaces, Istituto Italiano di Tecnologia, Pontedera, Italy, ${ }^{2}$ Department of Mechanical and Aerospace \\ Engineering, Politecnico di Torino, Torino, Italy
}

With the increasing advances in the fabrication and in monitoring approaches of nanotechnology devices, novel materials are being synthesized and tested for the interaction with biological environments. Among them, smart materials in particular provide versatile and dynamically tunable platforms for the investigation and manipulation of several biological activities with very low invasiveness in hardly accessible anatomical districts.

Edited by:

João Conde,

Massachusetts Institute of Technology, United States

Reviewed by:

Stefano Leporatti,

Istituto di Nanotecnologia

(CNR), Italy

Varpu Seija Marjomäki,

University of Jyväskylä, Finland

*Correspondence:

Giada Graziana Genchi giada.genchi@iit.it;

Gianni Ciofani

gianni.ciofani@iit.it

Specialty section:

This article was submitted

to Nanobiotechnology,

a section of the journal

Frontiers in Bioengineering

and Biotechnology

Received: 02 November 2017 Accepted: 05 December 2017

Published: 18 December 2017

Citation:

Genchi GG, Marino A, Tapeinos C and Ciofani $G$ (2017) Smart Materials

Meet Multifunctional Biomedical

Devices: Current and Prospective Implications for Nanomedicine.

Front. Bioeng. Biotechnol. 5:80. doi: 10.3389/fbioe.2017.00080
In the following, we will briefly recall recent examples of nanotechnology-based materials that can be remotely activated and controlled through different sources of energy, such as electromagnetic fields or ultrasounds, for their relevance to both basic science investigations and translational nanomedicine. Moreover, we will introduce some examples of hybrid materials showing mutually beneficial components for the development of multifunctional devices, able to simultaneously perform duties like imaging, tissue targeting, drug delivery, and redox state control. Finally, we will highlight challenging perspectives for the development of theranostic agents (merging diagnostic and therapeutic functionalities), underlining open questions for these smart nanotechnology-based devices to be made readily available to the patients in need.

Keywords: smart materials, remote stimulation, drug delivery, immune system, tissue engineering

\section{INTRODUCTION}

Smart materials have gained increasing attention in the biomedical research fields thanks to their adjustable physical and/or chemical properties in response to deliberately imparted external stimuli or to environmental changes. For these reasons, their introduction in nanomedicine has opened unprecedented possibilities of manipulation of biological entities at cellular and even sub-cellular level. In this scenario, the intrinsic properties of nanoparticles or nanotextured materials are exploited, providing active devices capable of diagnostic, therapeutic or even theranostic functions. When physical cues like light irradiation, ultrasounds, or electromagnetic fields are applied to a smart nanostructure, an energy transduction occurs and results into the activation of a precise cellular functionality. Moreover, a suitable modification of the nanoparticle surface (e.g., with the aid of a cell ligand or of a monoclonal antibody) can improve the efficacy of this activation, by targeting specific cell populations or even specific intracellular organelles. This approach, which can be defined as a new paradigm in nanomedicine, finds several applications including cancer therapy, drug delivery, tissue engineering, and even bionics. 
In this mini-review, we will focus on those nanomaterials that, in our opinion, are the most promising in terms of clinical translation, with particular attention to nanoparticles that act as "nano-transducers," allowing for a remote manipulation of biological activities, and thus providing a "smart" interface between biological and non-biological environments (Figure 1).

\section{REMOTE CELL STIMULATION THROUGH SMART NANOMATERIALS}

The possibility to finely and remotely manipulate cell behavior in deep tissues is of extreme importance in medicine for restoring physiological cell activities after the onset of a pathological condition (Dell'Anno et al., 2014; Li et al., 2017). Furthermore, the remote control of cell activities in vivo allows the elucidation of mechanisms at the base of different diseases and the development of novel therapeutic strategies (Brunoni et al., 2012; Paz et al., 2013; Legon et al., 2014).

A consolidated method for the fine modulation of the activity of specific cell types is represented by optogenetics, which consists in the genetic sensitization of targeted cells to light through a promoter-driven expression of light-sensitive proteins. Alternatively, a new generation of smart nanomaterial-based approaches for the remote control of cell behavior has recently been proposed (Genchi et al., 2017a). Smart nanomaterials can be externally/wirelessly activated by different energy sources [e.g., near-infrared (NIR) radiations, radiofrequency stimulations, magnetic fields, ultrasounds, etc.] that are able to penetrate biological tissues efficiently and non-invasively. Nanostructure activation in deep tissues triggers specific behaviors (e.g., neural spikes and myocyte contractions) (Eom et al., 2014; Colombo et al., 2016; Marino et al., 2017a), or tunes biochemical pathways involved in different cell activities, such as differentiation (Kim et al., 2016; Rau et al., 2016), morphological maturation (Ciofani et al., 2010), and hormone release (Stanley et al., 2012, 2015). These energy-driven nanoparticle-mediated approaches are able to overcome the scarce tissue penetration by visible light and the use of viruses to genetically modify target cells, which are the main drawbacks currently limiting clinical applications of optogenetics (Jarvis and Schultz, 2015).

Piezoelectric nanomaterials are a class of nanostructures able to generate a voltage on their surface when exposed to a mechanical stimulation, for example by means of ultrasounds, US (Wang et al., 2007). This voltage has been used for the stimulation of electrically excitable cells, like neurons (Ciofani et al., 2010; Royo-Gascon et al., 2013; Hoop et al., 2017; Lee et al., 2017) and bone cells (Genchi et al., 2017b). Our group demonstrated for the first time that the acute US-driven piezo-stimulation of barium titanate nanoparticles (BTNPs) associated to plasma membrane was able to significantly increase the intracellular calcium concentration in neural cells (Marino et al., 2015). The combination of US and non-piezoelectric BTNPs was not able to elicit a significant neural response, thus confirming that the mechanism was mediated by piezoelectricity and not by other unspecific phenomena (e.g., mechanical or thermal).

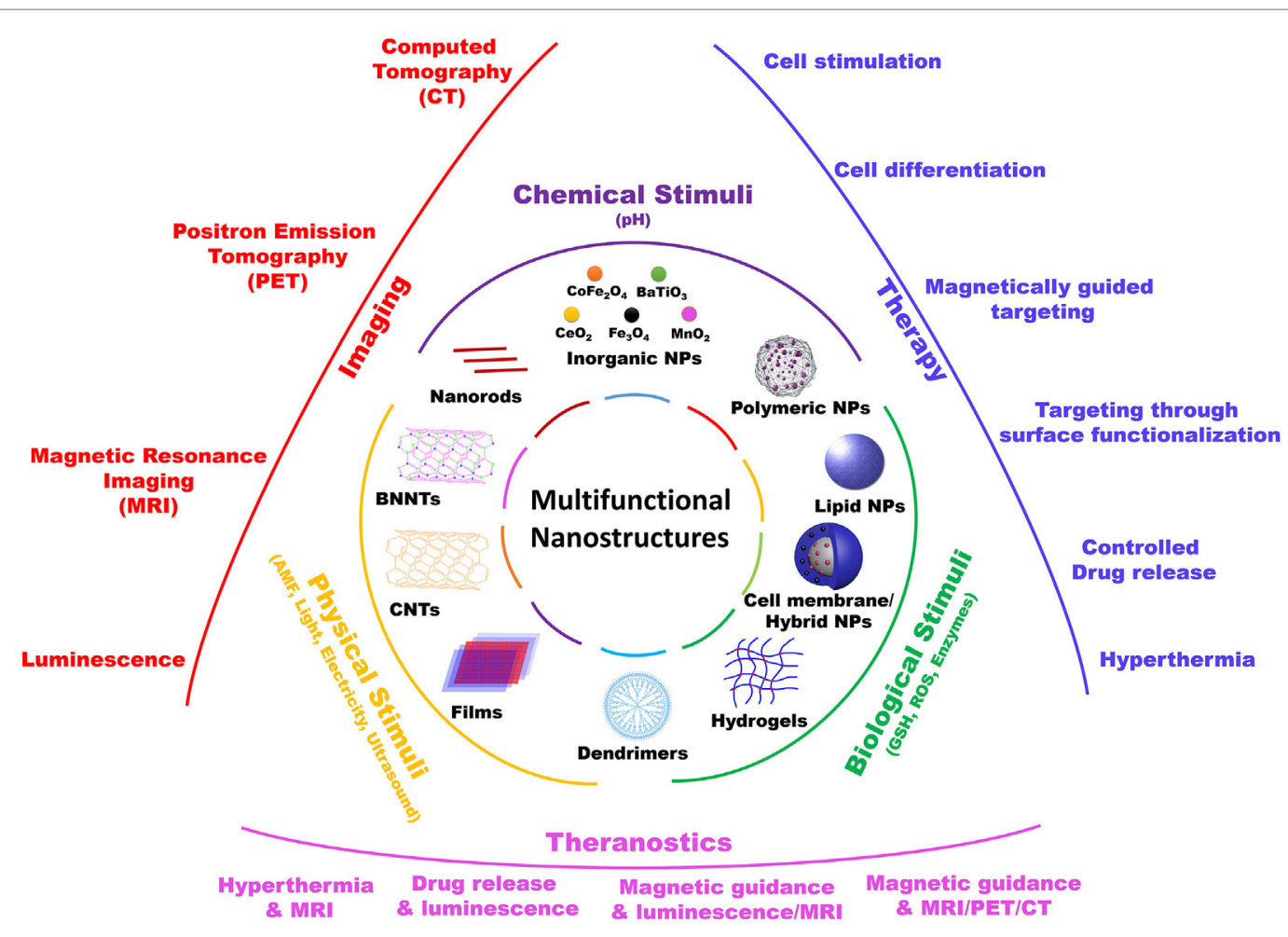

FIGURE 1 | Schematic representation of various multifunctional nanostructures, their responsive stimuli, and their main biomedical applications. 
Another wide-spread approach for remote cell activation is represented by nanoparticle-assisted heat stimulation by shortduration temperature increments in a physiological range (of about $5^{\circ} \mathrm{C}$ ) (Shapiro et al., 2012). A local increment of temperature can be obtained by exploiting different energy transduction approaches, such as the photothermal and the magnetothermal ones. Photothermal stimulation consists in the transduction of photon energy into heat and can be remotely triggered with NIR radiation in combination with many plasmonic nanomaterials, such as gold nanoshells (Erickson and Tunnell, 2010), gold nanorods (Huang et al., 2006), single-walled carbon nanotubes (Mocan et al., 2011), graphene oxide (Robinson et al., 2011), and copper sulfide $\left(\mathrm{Cu}_{2} \mathrm{~S}\right)$ nanocrystals (Wang et al., 2015). Different independent works showed that photothermal stimulation is able to reversibly elicit a neural response in terms of spike activity, intracellular calcium levels, and neurite outgrowth (Yong et al., 2014; Paviolo and Stoddart, 2017). These effects seem to be mediated by the opening of temperature-sensitive calcium channels (Miyako et al., 2014) and/or by heat-dependent capacitance changes of the neural plasma membrane (Carvalho-de-Souza et al., 2015).

However, the mechanisms of photothermal stimulation on complex neural networks have to be further investigated. Indeed, a recent work documented an inhibited neural network activity of hippocampal primary culture treated with gold nanorods upon NIR irradiation (Yoo et al., 2014). Remote photothermal nerve activation was successfully used for inducing leg muscle contraction in frogs after treatment with carbon nanohorns and NIR irradiation (Miyako et al., 2014). Similarly to neural cells, muscle cells can be stimulated by heat: in this concern, our group has recently demonstrated that an acute NIR irradiation of gold nanoshell-containing cultures is able to induce myotube contraction, while a chronic one suggested to promote mitochondriogenesis (Marino et al., 2017a).

Alternatively to photothermal stimulation, magnetothermal transduction can be exploited for cell heating/stimulation. In this case, magnetic nanoparticles dissipate heat when undergo an alternate electric field (Noh et al., 2017). In a recent work, this approach was exploited for remote deep stimulation of the ventral tegmental area (VTA) through the opening of a transfected heat-sensitive receptor TRPV1 in mice. The consequent increase of neural activity was also observed in the brain areas receiving excitatory projections from VTA. Interestingly, the retention period of the magnetic nanoparticles in the VTA was longer than a month, thus allowing for chronic magnetothermal VTA stimulations (Chen et al., 2015).

Other nanomaterial-assisted remote stimulation methods exploit magnetoelectric and optoelectric phenomena (Colombo et al., 2016; Wang and Guo, 2016). Concerning magnetoelectric materials, cobalt ferrite-barium titanate $\left(\mathrm{CoFe}_{2} \mathrm{O}_{4}-\mathrm{BaTiO}_{3}\right)$ coreshell nanoparticles were used to modulate deep-brain activity under a low-intensity alternating magnetic field (Guduru et al., 2015). Moreover, the magnetic properties of these nanoparticles also facilitated their delivery to the central nervous system (CNS). Indeed, $\mathrm{CoFe}_{2} \mathrm{O}_{4}-\mathrm{BaTiO}_{3}$ nanoparticles were intravenously administered in mouse tail and forced to cross the blood-brain barrier (BBB) with a static magnetic field.
Regarding the frontiers of optoelectronic bio-interfaces, polymeric biocompatible organic nanoparticles/films of poly(3-hexylthiophene) revealed great performances both in restoring the functionality of blind retinas on ex vivo models [i.e., explants of rat retinas characterized by photoreceptor degeneration (Ghezzi et al., 2013)], and in modulating the behavior of eyeless animals (i.e., freshwater polyps) by amplifying the function of their primitive photoreceptors (Tortiglione et al., 2017).

As a further example, a class of nanomaterials characterized by elements with high atomic number $Z$ (e.g., Au/Pt nanoparticles) can be also exploited as contrast agents and sensitizers of X-rays, representing very promising nanovectors for cancer theranostics (Subiel et al., 2016). The great versatility and potential of these nanomaterials in nanomedicine is noteworthy, especially considering their great potential also in proton therapy (Schlatholter et al., 2016).

\section{MULTIFUNCTIONAL DRUG DELIVERY SYSTEMS (MDDS)}

The optimization of carriers for delivering drugs specifically to diseased areas originated from the need to overcome drug limitations, such as cytotoxicity, immunogenicity, short circulation times, non-controlled bio-distribution, and the non-targeting ability toward specific tissues. During recent years, significant advancements in the field of nanobiotechnology led to the development of MDDS for the accomplishment of diagnostic and therapeutic purposes through a single medical device, able of performing bio-imaging duties, while improving the therapeutic efficacy of drugs available on the market. MDDS already presented in the literature can have a variety of morphologies and sizes, and can be made of various materials like natural and synthetic polymers, lipids, and other inorganic and organic materials. Nanostructures made of polymeric materials are the most used MDDS, due to a number of advantages, including the high versatility during their fabrication, their controllable size and shape, their high encapsulation efficiency, and their easy surface functionalization by numerous targeting groups, including peptides, proteins, and antibodies. Furthermore, coating of these nanostructures with stealth materials like poly(ethylene glycol), PEG, increases their circulation time inside the body. In combination with the targeting ability, this renders them suitable for the specific delivery of a number of therapeutic preparations, including drugs, enzymes, proteins, RNA, and DNA (Mura et al., 2013; Srinivasan et al., 2015; Bose et al., 2016; Lu et al., 2016) to specific diseased tissues. The most used polymeric nanostructures are nanoparticles, nanocapsules, dendrimers, nanospheres, spherical and worm-like micelles, nanotubes, and hydrogels (Theato et al., 2013; Torchilin, 2014; Lu et al., 2016).

Another category of nanomaterials used as MDDS is represented by lipid nanostructures, including niosomes, transfersomes, liposomes, solid lipid nanoparticles, and nanostructured lipid carriers. Lipid-based nanostructures are considered a better alternative compared to polymeric nanostructures, especially for the treatment of CNS diseases, due to their inherent biocompatibility, 
low immunogenicity, and their ability to penetrate the BBB. A newer approach, similar to that of lipid-based nanostructures, but more biomimetic, relies on cell membrane-derived vesicles or the coating of existing nanoparticles such as poly(lactide-coglycolide) and magnetite nanoparticles with the cell membrane of various cells, like macrophages, neutrophils, red blood cells, cancer cells, and others (Fang et al., 2014; Luk and Zhang, 2015; Bose et al., 2016; Gao et al., 2016; Krishnamurthy et al., 2016).

Inorganic nano- and microparticles, including magnetite $\left(\mathrm{Fe}_{3} \mathrm{O}_{4}\right)$, maghemite $\left(\gamma-\mathrm{Fe}_{2} \mathrm{O}_{3}\right)$, manganese dioxide $\left(\mathrm{MnO}_{2}\right)$, cerium dioxide $\left(\mathrm{CeO}_{2}\right)$, platinum $(\mathrm{Pt})$, silver $(\mathrm{Ag})$, zinc oxide $\left(\mathrm{ZnO}_{2}\right)$, silica $\left(\mathrm{SiO}_{2}\right)$, titanium dioxide $\left(\mathrm{TiO}_{2}\right)$, and others, have also been used as MDDS combining various functionalities, such as imaging and hyperthermia (Hayashi et al., 2013; Tapeinos et al., 2016), reactive oxygen species (ROS) scavenging and oxygen generation (Bizeau et al., 2017; Tapeinos et al., 2017), and antimicrobial and anticancer activities (Mohanta et al., 2017).

A combination of the above-mentioned structures like polymers and lipids, polymers and inorganic nanoparticles, and lipids and inorganic nanoparticles have also been studied aiming at further improving the multifunctionality and the therapeutic effect of these nanostructures. Our group, for instance, used a lipid matrix to encapsulate iron oxide nanoparticles and the drug sorafenib, and subsequently showed its magnetically-driven accumulation in vitro against hepatocarcinoma cells, demonstrating localized therapy (Grillone et al., 2015). A combination of inorganic and polymeric nanoparticles have also been used for multimodal imaging of breast cancer tissue (Cheng et al., 2012), providing a more precise imaging through luminescence and magnetic resonance.

The multifunctionality of each one of the above-mentioned nanostructures derives from the combination of materials with specific characteristics and can be translated as the response of the MDDS to a physical/external and/or a biological/internal stimulus, by changing their morphological (size, shape) and/ or their physicochemical characteristics (colloidal stability, crystal structure, hydrophobicity, redox state, etc.). To date, numerous systems that respond to external and/or internal stimuli have been developed, but the most important are those ones that respond to more than one stimulus and show ability of a most precise control in the release of the encapsulated therapeutic molecules. Stimuli-responsive systems are usually a combination of polymeric nanostructures and inorganic nanoparticles (Tapeinos et al., 2013, 2016, 2017), although lipid-based nanostructures have also been developed to alter their properties when an external stimulus is applied (Du et al., 2015).

Stimuli-responsive nanostructures that have been used for the treatment of various diseases can respond to physical stimuli, including ultrasounds, light, electric fields, and magnetic fields (Mura et al., 2013; Marino et al., 2015, 2017b,c; Genchi et al., $2016,2017 b)$, or they can take advantage of the changes in the biological microenvironment of each disease and respond to alterations in temperature, $\mathrm{pH}$, redox conditions, $\mathrm{ROS}$, and enzyme concentration (Tapeinos et al., 2008, 2017; de la Rica et al., 2012; Torchilin, 2014; Tapeinos and Pandit, 2016; Bizeau et al., 2017). Furthermore, a combination of these stimuli (Mura et al., 2013;
Tapeinos et al., 2013, 2016; Efthimiadou et al., 2014a,b; Lu et al., 2016) have also been used to increase the therapeutic versatility of the MDDS.

\section{APPLICATIONS AND FUTURE PERSPECTIVES}

An increasing number of nanotechnology-based strategies are available for wireless and low-invasiveness manipulation of biological activities in hardly accessible anatomical districts, as the CNS. Among them, ultrasound activation of piezoelectric materials is highly promising for the treatment of deep tissues (Tufail et al., 2011). Further investigations are, however, necessary for selective delivery of piezoelectric nanomaterials to target tissues and retention on site in vivo. The first studies on toxicity and accumulation of piezoelectric boron nitride nanotubes was conducted by our group with rabbits. Our studies collectively demonstrated high biocompatibility in terms of liver, kidney, and blood parameters of high doses (up to $10 \mathrm{mg} / \mathrm{kg}$ ) of intravenously injected nanotubes (Ciofani et al., 2012, 2013a).

Our group also provided the first evidences on the applicability of piezoelectric nanocomposite films based on poly(vinylidenefluoride-co-trifluoroethylene), $\mathrm{P}(\mathrm{VDF}-\mathrm{TrFE})$, and BTNPs to neuron-like cell stimulation for cochlear prosthetics. Aiming at compensating missing/altered hair cell function, our nanocomposite films were satisfactorily tested for stimulation of a human neuronal model by direct piezoelectric effect (Genchi et al., 2016). A single US application to films supporting SH-SY5Y cell cultures triggered significantly higher calcium influxes than plain $\mathrm{P}(\mathrm{VDF}-\mathrm{TrFE})$ films and non-piezoelectric control substrates. Repeated stimulations significantly increased expression of $\beta 3$-tubulin and neurite extension due to the better piezoelectric properties of composite films, suggesting improved functional maturation of the neuronal model on our artificial cochlear epithelium.

Smart nanomaterials are providing concrete opportunities for the treatment of pathological conditions that affect deep anatomical districts. However, hybrid smart material devices are opening even unprecedented therapeutic opportunities in nanomedicine, like biological barrier overcoming while multiple functions are accomplished (imaging, drug release, etc.). In particular, low-intensity magnetic field stimulation of magnetoelectric nanomaterials holds promise of clinical practice application in the near future. For instance, $\mathrm{CoFe}_{2} \mathrm{O}_{4}-\mathrm{BaTiO}_{3}$ were validated for Parkinson's disease treatment in silico (Yue et al., 2012). Moreover, they were successfully tested for magnetically driven BBB crossing, as well as for coupling with and mapping the intrinsic neural activity in mice (Guduru et al., 2015). $\mathrm{CoFe}_{2} \mathrm{O}_{4}-\mathrm{BaTiO}_{3}$ nanoparticles were also used for direct current-field cell targeting and alternating current-field releasing of anti-HIV drug in vitro (Nair et al., 2013) and of paclitaxel for ovarian cancer treatment in vivo (Rodzinski et al., 2016).

Highly encouraging to the treatment of deep tissues are also cerium dioxide nanomaterials, alone and in combinations with other materials for the control of redox environments (both intercellular and intracellular ones). Our group demonstrated 
TABLE 1 | Stimuli and effects of various multifunctional responsive structures.

\begin{tabular}{|c|c|c|c|}
\hline Responsive material & Stimulus & Effect & Reference \\
\hline Au nanorods & Photothermal (NIR radiation) & Neuronal spikes & Eom et al. (2014) \\
\hline $\mathrm{Au} @ \mathrm{SiO}_{2}$ nanoshells & Photothermal (NIR radiation) & Myotube contractions & Marino et al. (2017a) \\
\hline $\begin{array}{l}\text { Peptide ligands assembled on carbon } \\
\text { nanotubes }\end{array}$ & Light & Cell differentiation & Kim et al. (2016) \\
\hline Au nanoparticles & Photothermal & Cell differentiation & Rau et al. (2016) \\
\hline $\mathrm{Cu}_{2} \mathrm{~S}$ nanocrystals & $\begin{array}{l}\text { Photothermal and photodynamic } \\
\text { (NIR radiation) }\end{array}$ & Hyperthermia and ROS-induced apoptosis & Wang et al. (2015) \\
\hline Au nanorods & Photothermal (NIR radiation) & Cancer cell imaging and photothermal therapy & Huang et al. (2006) \\
\hline Au nanorods & Photothermal (NIR radiation) & Inhibition of spontaneous and epileptiform neural activity & Yoo et al. (2014) \\
\hline Au nanorods & Photothermal (NIR radiation) & Evoking spikes on primary auditory neurons & Yong et al. (2014) \\
\hline Carbon nanohorns & Photothermal (NIR radiation) & $\begin{array}{l}\text { Nerve activation (opening of the temperature-sensitive } \\
\text { calcium channels) }\end{array}$ & Miyako et al. (2014) \\
\hline Ultrasmall reduced graphene oxide & NIR radiation & Photoablation of U87MG cancer cells & Robinson et al. (2011) \\
\hline ZnO nanowires & Ultrasounds & Continuous direct-current output & Wang et al. (2007) \\
\hline BNNTs & Ultrasounds & Neural stimulation (neurite outgrowth) & Ciofani et al. (2010) \\
\hline $\begin{array}{l}\mathrm{BaTiO}_{3} \text { nanoparticles with tetragonal } \\
\text { crystal }\end{array}$ & Ultrasounds & Neural stimulation (calcium and sodium waves) & Marino et al. (2015) \\
\hline PVDF film & Mechanical vibration & Neural stimulation (neurite outgrowth) & Royo-Gascon et al. (2013) \\
\hline PVDF membranes & Ultrasounds & Neural differentiation & Hoop et al. (2017) \\
\hline P(VDF-TrFE) conduits & N/A & Regeneration of transected adult rat spinal cord & Lee et al. (2017) \\
\hline P(VDF-TrFE)/BTNP composite films & Ultrasounds & $\begin{array}{l}\text { 1. Stimulation of a human neuronal model } \\
\text { 2. Increased calcium influx } \\
\text { 3. Increased expression of } \beta 3 \text {-tubulin } \\
\text { 4. Neurite extension }\end{array}$ & Genchi et al. (2016) \\
\hline P(VDF-TrFE)/BNNT composite films & Ultrasounds & Osteogenic differentiation & Genchi et al. (2017b) \\
\hline High-Z nanomaterials & lonizing radiations & Enhancement of irradiation effect & $\begin{array}{l}\text { Schlatholter et al. (2016); Subiel } \\
\text { et al. (2016) }\end{array}$ \\
\hline $\mathrm{Fe}_{3} \mathrm{O}_{4}$ magnetic nanoparticles & Magnetic field & $\begin{array}{l}\text { Deep stimulation of the ventral tegmental area through } \\
\text { opening of the transfected heat-sensitive receptor TRPV1 }\end{array}$ & Chen et al. (2015) \\
\hline $\mathrm{CeO}_{2}$ & ROS concentration & ROS scavenging & $\begin{array}{l}\text { Ciofani et al. (2013b, 2014); } \\
\text { Rocca et al. (2014, 2015) }\end{array}$ \\
\hline \multirow[t]{3}{*}{$\mathrm{CoFe}_{2} \mathrm{O}_{4}-\mathrm{BaTiO}_{3}$} & Ultrasounds/static magnetic field & Magnetically guided targeting & Yue et al. (2012) \\
\hline & Direct current-field & Cell targeting & \multirow{2}{*}{$\begin{array}{l}\text { Nair et al. (2013); Rodzinski } \\
\text { et al. (2016) }\end{array}$} \\
\hline & Alternating current-field & Drug release & \\
\hline $\mathrm{Fe}_{3} \mathrm{O}_{4}, \gamma-\mathrm{Fe}_{2} \mathrm{O}_{3}$ & Alternating magnetic field & $\begin{array}{l}\text { 1. Magnetic resonance imaging } \\
\text { 2. Controlled release } \\
\text { 3. Tumor reduction }\end{array}$ & $\begin{array}{l}\text { Hayashi et al. (2013); Tapeinos } \\
\text { et al. (2016) }\end{array}$ \\
\hline $\mathrm{MnO}_{2}$ & ROS concentration & ROS scavenging and oxygen generation & $\begin{array}{l}\text { Bizeau et al. (2017); Tapeinos } \\
\text { et al. (2017) }\end{array}$ \\
\hline $\mathrm{CoFe}_{2} \mathrm{O}_{4}-\mathrm{BaTiO}_{3}$ & $\begin{array}{l}\text { Low-intensity alternating magnetic } \\
\text { field/static magnetic field }\end{array}$ & Modulation of deep-brain activity/guided brain targeting & Guduru et al. (2015) \\
\hline \multirow[t]{2}{*}{ Poly(3-hexylthiophene) } & \multirow[t]{2}{*}{ Light } & Restoration of the functionality of blind retinas & Ghezzi et al. (2013) \\
\hline & & Modulation of the behavior of eyeless animals & Tortiglione et al. (2017) \\
\hline $\begin{array}{l}\beta \text {-cyclodextrin } / \mathrm{CeO}_{2} \\
\text { Ferrocene } / \mathrm{SiO}_{2}\end{array}$ & ROS concentration & $\begin{array}{l}\text { Intracellular compartment targeting, enhanced } \\
\text { antitumoral activity and drug delivery }\end{array}$ & Xu et al. (2013) \\
\hline $\begin{array}{l}\text { Poly (acrylic acid), } \mathrm{Fe}_{3} \mathrm{O}_{4}, \mathrm{Au}, \mathrm{NaYF}_{4} \text { : } \\
\mathrm{Yb}, \mathrm{Er}\end{array}$ & Magnetic field NIR irradiation & $\begin{array}{l}\text { 1. Multimodal imaging of breast cancer tissue } \\
\text { 2. Magnetically targeted photothermal therapy }\end{array}$ & Cheng et al. (2012) \\
\hline Cetyl palmitate/ $/ \mathrm{Fe}_{3} \mathrm{O}_{4}$ & Static magnetic field & Localized anticancer therapy & Grillone et al. (2015) \\
\hline DPPC, DSPE-PEG 2000 -folate, & Radiofrequency & 1. Magnetic resonance imaging & Du et al. (2015) \\
\hline $\mathrm{C} 60-\mathrm{Fe}_{3} \mathrm{O}_{4}-\mathrm{PEG}_{2000}$ & & $\begin{array}{l}\text { 2. Photothermal ablation } \\
\text { 3. Controlled release }\end{array}$ & \\
\hline \multirow[t]{4}{*}{ DMAEMA, AA, Disulfide, $\mathrm{Fe}_{3} \mathrm{O}_{4}$} & Alternating magnetic field & \multirow[t]{4}{*}{ Enhanced release of encapsulated anticancer drugs } & \multirow[t]{4}{*}{ Tapeinos et al. $(2013,2016)$} \\
\hline & Temperature & & \\
\hline & $\mathrm{pH}$ & & \\
\hline & GSH concentration & & \\
\hline \multirow[t]{2}{*}{ Carbon-based } & NIR irradiation & Stem-cell differentiation & Kim et al. (2016) \\
\hline & Laser Irradiation & Photothermal ablation & Mocan et al. (2011) \\
\hline Cell membrane of various cells, like & $\mathrm{N} / \mathrm{A}$ & 1. Tumor-specific immune response & Fang et al. (2014); Luk and \\
\hline macrophages, neutrophils, red blood & & 2. Specific targeting & Zhang (2015); Bose et al. \\
\hline cells, cancer cells & & 3. Drug delivery & $\begin{array}{l}\text { (2016); Gao et al. (2016); } \\
\text { Krishnamurthy et al. (2016) }\end{array}$ \\
\hline $\begin{array}{l}\text { Iron oxide nanoparticles and } \\
\text { genetically encoded ferritine } \\
\text { nanoparticles }\end{array}$ & Radiowave heating & Insulin transgene expression and proinsulin release & Stanley et al. $(2012,2015)$ \\
\hline
\end{tabular}


TABLE 1 | Continued

\begin{tabular}{llll}
\hline Responsive material & Stimulus & Effect & Reference \\
\hline Au nanoparticles & Photothermal (light at $532 \mathrm{~nm})$ & $\begin{array}{l}\text { Evoking spikes to hippocampal neurons through heat- } \\
\text { dependent changes of capacitance of the neural plasma } \\
\text { membrane }\end{array}$ & Carvalho-de-Souza et al. (2015) \\
& &
\end{tabular}

AA, acrylic acid; BNNTs, boron nitride nanotubes; BTNPs, barium titanate nanoparticles; C60, fullerene; Disulfide, N,N'-(disulfanediylbis (ethane-2,1-diyl))bis(2-methylacrylamide); DMAEMA, dimethyl aminoethyl methacrylate; DPPC, dipalmitoylphosphatidylcholine; DSPE-PEG ${ }_{2000}$-folate, 1,2-distearoyl-sn-glycero-3-phospho-ethanolamine-N-[carboxy (poly(ethylene glycol))-2000]-folate; GSH, glutathione; NIR, near infrared; PEG, poly(ethylene glycol); PVDF, poly(vinylidenefluoride); P(VDF-TrFE), poly(vinylidenefluoride-cotrifluoroethylene); ROS, reactive oxygen species.

suitability of $\mathrm{CeO}_{2}$ nanoparticles to ROS scavenging in vitro and in vivo, with relevant findings to Parkinson's disease (Ciofani et al., 2013b, 2014) and obesity treatment (Rocca et al., 2014, 2015). However, the great potentialities of nanoceria can find full exploitation by targeting specific cell populations and intracellular anatomic districts. A first example of multifunctional platform based on $\mathrm{CeO}_{2}$ nanoparticles involved coating with $\beta$-cyclodextrin and association to ferrocene-mesoporous $\mathrm{SiO}_{2}$ nanoparticles, MSN (Xu et al., 2013). These hybrid nanoparticles promoted HEK293 normal cell proliferation, while they limited that one of A549 cancer cells. Moreover, they mostly localized in the cytosol of HEK293, whereas in the lysosomes of A549. In these acidic organelles, the nanoparticles underwent dissolution and exerted a strong cytotoxic effect by the synergic action of $\mathrm{CeO}_{2}$ nanoparticles and oxidized ferrocene. This anticancer activity was even increased when the MSN pores were loaded with floxuridine as a antitumor drug model. In this way, $\mathrm{CeO}_{2}-$ based nanoparticles accomplished at the same time intracellular compartment targeting, antitumoral activity, and drug delivery functions.

Other interesting multifunctional materials responsive to external stimuli are halloysite nanotubes (HNTs), that for instance were used for the preparation of temperatureresponsive platforms by poly( $N$-isopropylacrylamide) coating and curcumin loading, for subsequent release upon temperature increase (Cavallaro et al., 2015). In particular, the platforms were tested in acidic environment simulating the gastrointestinal transit and exerted successful protection/delivery of their curcumin payload with promising implications in cancer treatment. In another study, HNTs were coated with folate via a complex redox-responsive disulfide bond and used for targeting/release of doxorubicin in a reducing environment in vitro, also providing safe drug delivery to tumor sites in nude mice (Hu et al., 2017).

\section{REFERENCES}

Bizeau, J., Tapeinos, C., Marella, C., Larrañaga, A., and Pandit, A. (2017). Synthesis and characterization of hyaluronic acid coated manganese dioxide microparticles that act as ROS scavengers. Colloids Surf. B Biointerfaces 159, 30-38. doi:10.1016/j.colsurfb.2017.07.081

Bose, R. J., Lee, S. H., and Park, H. (2016). Biofunctionalized nanoparticles: an emerging drug delivery platform for various disease treatments. Drug Discov. Today 21, 1303-1312. doi:10.1016/j.drudis.2016.06.005

Brunoni, A. R., Nitsche, M. A., Bolognini, N., Bikson, M., Wagner, T., Merabet, L., et al. (2012). Clinical research with transcranial direct current stimulation (tDCS): challenges and future directions. Brain Stimul. 5, 175-195. doi:10.1016/j. brs.2011.03.002
A detailed list of multifunctional nanostructures, their responsive stimuli, and their biological effects can be found in Table 1.

\section{CONCLUSION}

To date, numerous studies have reported the enhanced therapeutic effect of several nanostructured devices compared to traditional systems. However, a lot of work is still mandatory for these materials to provide a solution to unmet clinical needs. Non-invasive approaches for remote targeting and activation are nowadays available, yet the development of complex and reliable models of biological barriers in vitro and in vivo is necessary to ensure proper device testing. In our opinion, the enhancement of the capability of intracellular compartment targeting by fine tuning/control of the surface physicochemical properties represents a key step for these smart materials to be made available to the next future clinical practice.

\section{AUTHOR CONTRIBUTIONS}

GC wrote the Section "Introduction," GG wrote the Sections "Applications and Future Perspectives" and "Conclusion," AM wrote the "Remote cell stimulation through smart nanoparticles" Section, and CT wrote the "Multifunctional drug delivery systems" Section and prepared Figure and Table. GC revised the whole manuscript.

\section{FUNDING}

The preparation of this manuscript was partially supported by the Italian Ministry of Health Grant Number RF-2011-02350464, by the Agenzia Spaziale Italiana Contract Number 2016-7-U.0 (NANOROS), by the European Research Council Grant Number 709613 (SLaMM), by the Compagnia di San Paolo Starting Grant Number 55_AI16GC01, and by Fondazione Umberto Veronesi.

Carvalho-de-Souza, J. L., Treger, J. S., Dang, B., Kent, S. B., Pepperberg, D. R., and Bezanilla, F. (2015). Photosensitivity of neurons enabled by celltargeted gold nanoparticles. Neuron 86, 207-217. doi:10.1016/j.neuron.2015. 02.033

Cavallaro, G., Lazzara, G., Massaro, M., Milioto, S., Noto, R., Parisi, F., et al. (2015). Biocompatible Poly(N-isopropylacrylamide)-halloysite nanotubes for thermoresponsive curcumin release. J. Phys. Chem. C 119, 8944-8951. doi:10.1021/ acs.jpcc.5b00991

Chen, R., Romero, G., Christiansen, M. G., Mohr, A., and Anikeeva, P. (2015). Wireless magnetothermal deep brain stimulation. Science 347, 1477-1480. doi:10.1126/science.1261821

Cheng, L., Yang, K., Li, Y., Zeng, X., Shao, M., Lee, S. T., et al. (2012). Multifunctional nanoparticles for upconversion luminescence/MR multimodal imaging and 
magnetically targeted photothermal therapy. Biomaterials 33, 2215-2222. doi:10.1016/j.biomaterials.2011.11.069

Ciofani, G., Danti, S., D’Alessandro, D., Ricotti, L., Moscato, S., Bertoni, G., et al. (2010). Enhancement of neurite outgrowth in neuronal-like cells following boron nitride nanotube-mediated stimulation. ACS Nano 4, 6267-6277. doi:10.1021/nn101985a

Ciofani, G., Danti, S., Genchi, G. G., D’Alessandro, D., Pellequer, J. L., Odorico, M., et al. (2012). Pilot in vivo toxicological investigation of boron nitride nanotubes. Int. J. Nanomedicine 7, 19-24. doi:10.2147/IJN.S28144

Ciofani, G., Danti, S., Nitti, S., Mazzolai, B., Mattoli, V., and Giorgi, M. (2013a). Biocompatibility of boron nitride nanotubes: an up-date of in vivo toxicological investigation. Int. J. Pharm. 444, 85-88. doi:10.1016/j.ijpharm.2013.01.037

Ciofani, G., Genchi, G. G., Liakos, I., Cappello, V., Gemmi, M., Athanassiou, A., et al. (2013b). Effects of cerium oxide nanoparticles on PC12 neuronal-like cells: proliferation, differentiation, and dopamine secretion. Pharm. Res. 30, 2133-2145. doi:10.1007/s11095-013-1071-y

Ciofani, G., Genchi, G. G., Mazzolai, B., and Mattoli, V. (2014). Transcriptional profile of genes involved in oxidative stress and antioxidant defense in PC12 cells following treatment with cerium oxide nanoparticles. Biochim. Biophys. Acta 1840, 495-506. doi:10.1016/j.bbagen.2013.10.009

Colombo, E., Feyen, P., Antognazza, M. R., Lanzani, G., and Benfenati, F. (2016). Nanoparticles: a challenging vehicle for neural stimulation. Front. Neurosci. 10:105. doi:10.3389/fnins.2016.00105

de la Rica, R., Aili, D., and Stevens, M. M. (2012). Enzyme-responsive nanoparticles for drug release and diagnostics. Adv. Drug Deliv. Rev. 64, 967-978. doi:10.1016/j.addr.2012.01.002

Dell'Anno, M. T., Caiazzo, M., Leo, D., Dvoretskova, E., Medrihan, L., Colasante, G., et al. (2014). Remote control of induced dopaminergic neurons in parkinsonian rats. J. Clin. Invest. 124, 3215-3229. doi:10.1172/JCI74664

Du, B., Han, S., Li, H., Zhao, F., Su, X., Cao, X., et al. (2015). Multi-functional liposomes showing radiofrequency-triggered release and magnetic resonance imaging for tumor multi-mechanism therapy. Nanoscale 7, 5411-5426. doi:10.1039/c4nr04257c

Efthimiadou, E. K., Tapeinos, C., Chatzipavlidis, A., Boukos, N., Fragogeorgi, E., Palamaris, L., et al. (2014a). Dynamic in vivo imaging of dual-triggered microspheres for sustained release applications: synthesis, characterization and cytotoxicity study. Int. J. Pharm. 461, 54-63. doi:10.1016/j.ijpharm.2013. 11.037

Efthimiadou, E. K., Tapeinos, C., Tziveleka, L. A., Boukos, N., and Kordas, G. (2014b). PH- and thermo-responsive microcontainers as potential drug delivery systems: morphological characteristic, release and cytotoxicity studies. Mater. Sci. Eng. C 37, 271-277. doi:10.1016/j.msec.2014.01.024

Eom, K., Kim, J., Choi, J. M., Kang, T., Chang, J. W., Byun, K. M., et al. (2014). Enhanced infrared neural stimulation using localized surface plasmon resonance of gold nanorods. Small 10, 3853-3857. doi:10.1002/smll.201400599

Erickson, T. A., and Tunnell, J. W. Gold Nanoshells in Biomedical Applications. Weinheim: Wiley-VCH (2010).

Fang, R. H., Hu, C. M., Luk, B. T., Gao, W., Copp, J. A., Tai, Y., et al. (2014). Cancer cell membrane-coated nanoparticles for anticancer vaccination and drug delivery. Nano Lett. 14, 2181-2188. doi:10.1021/nl500618u

Gao, C., Lin, Z., Jurado-Sanchez, B., Lin, X., Wu, Z., and He, Q. (2016). Stem cell membrane-coated nanogels for highly efficient in vivo tumor targeted drug delivery. Small 12, 4056-4062. doi:10.1002/smll.201600624

Genchi, G. G., Ceseracciu, L., Marino, A., Labardi, M., Marras, S., Pignatelli, F., et al. (2016). $\mathrm{P}(\mathrm{VDF}-\mathrm{TrFE}) / \mathrm{BaTiO} 3$ nanoparticle composite films mediate piezoelectric stimulation and promote differentiation of SH-SY5Y neuroblastoma cells. Adv. Healthc. Mater. 5, 1808-1820. doi:10.1002/adhm.201600245

Genchi, G. G., Marino, A., Grillone, A., Pezzini, I., and Ciofani, G. (2017a). Remote control of cellular functions: The role of smart nanomaterials in the medicine of the future. Adv. Healthc. Mater. 6, 1700002. doi:10.1002/adhm.201700002

Genchi, G. G., Sinibaldi, E., Ceseracciu, L., Labardi, M., Marino, A., Marras, S., et al. (2017b). Ultrasound-activated piezoelectric $\mathrm{P}(\mathrm{VDF}-\mathrm{TrFE}) / \mathrm{boron}$ nitride nanotube composite films promote differentiation of human SaOS-2 osteoblast-like cells. Nanomedicine. doi:10.1016/j.nano.2017.05.006

Ghezzi, D., Antognazza, M. R., Maccarone, R., Bellani, S., Lanzarini, E., Martino, N., et al. (2013). A polymer optoelectronic interface restores light sensitivity in blind rat retinas. Nat. Photonics 7, 400-406. doi:10.1038/nphoton.2013.34

Grillone, A., Riva, E. R., Mondini, A., Forte, C., Calucci, L., Innocenti, C., et al. (2015). Active targeting of sorafenib: preparation, characterization, and in vitro testing of drug-loaded magnetic solid lipid nanoparticles. Adv. Healthc. Mater. 4, 1681-1690. doi:10.1002/adhm.201500235

Guduru, R., Liang, P., Hong, J., Rodzinski, A., Hadjikhani, A., Horstmyer, J., et al. (2015). Magnetoelectric 'spin' on stimulating the brain. Nanomedicine (Lond) 10, 2051-2061. doi:10.2217/nnm.15.52

Hayashi, K., Nakamura, M., Sakamoto, W., Yogo, T., Miki, H., Ozaki, S., et al. (2013). Superparamagnetic nanoparticle clusters for cancer theranostics combining magnetic resonance imaging and hyperthermia treatment. Theranostics 3, 366-376. doi:10.7150/thno.5860

Hoop, M., Chen, X. Z., Ferrari, A., Mushtaq, F., Ghazaryan, G., Tervoort, T., et al. (2017). Ultrasound-mediated piezoelectric differentiation of neuron-like PC12 cells on PVDF membranes. Sci. Rep. 7, 4028. doi:10.1038/ s41598-017-03992-3

Hu, Y., Chen, J., Li, X., Sun, Y., Huang, S., Li, Y., et al. (2017). Multifunctional halloysite nanotubes for targeted delivery and controlled release of doxorubicin in-vitro and in-vivo studies. Nanotechnology 28, 375101. doi:10.1088/ 1361-6528/aa8393

Huang, X., El-Sayed, I. H., Qian, W., and El-Sayed, M. A. (2006). Cancer cell imaging and photothermal therapy in the near-infrared region by using gold nanorods. J. Am. Chem. Soc. 128, 2115-2120. doi:10.1021/ja057254a

Jarvis, S., and Schultz, S. R. (2015). Prospects for optogenetic augmentation of brain function. Front. Syst. Neurosci. 9:157. doi:10.3389/fnsys.2015.00157

Kim, H. W., Yang, K., Jeong, W. J., Choi, S. J., Lee, J. S., Cho, A. N., et al. (2016). Photoactivation of noncovalently assembled peptide ligands on carbon nanotubes enables the dynamic regulation of stem cell differentiation. ACS Appl. Mater. Interfaces 8, 26470-26481. doi:10.1021/acsami.6b06796

Krishnamurthy, S., Gnanasammandhan, M. K., Xie, C., Huang, K., Cui, M. Y., and Chan, J. M. (2016). Monocyte cell membrane-derived nanoghosts for targeted cancer therapy. Nanoscale 8, 6981-6985. doi:10.1039/c5nr07588b

Lee, Y. S., Wu, S., Arinzeh, T. L., and Bunge, M. B. (2017). Enhanced noradrenergic axon regeneration into Schwann cell-filled PVDF-TrFE conduits after complete spinal cord transection. Biotechnol. Bioeng. 114, 444-456. doi:10.1002/ bit.26088

Legon, W., Sato, T. F., Opitz, A., Mueller, J., Barbour, A., Williams, A., et al. (2014). Transcranial focused ultrasound modulates the activity of primary somatosensory cortex in humans. Nat. Neurosci. 17, 322-329. doi:10.1038/ nn. 3620

Li, J., Liu, J., and Chen, C. (2017). Remote control and modulation of cellular events by plasmonic gold nanoparticles: implications and opportunities for biomedical applications. ACS Nano 11, 2403-2409. doi:10.1021/acsnano.7b01200

Lu, Y., Aimetti, A. A., Langer, R., and Gu, Z. (2016). Bioresponsive materials. Nat. Rev. Mater. 2, 16075. doi:10.1038/natrevmats.2016.75

Luk, B. T., and Zhang, L. (2015). Cell membrane-camouflaged nanoparticles for drug delivery. J. Control Release 220(Pt B), 600-607. doi:10.1016/j.jconrel. 2015.07.019

Marino, A., Arai, S., Hou, Y., Degl'Innocenti, A., Cappello, V., Mazzolai, B., et al. (2017a). Gold nanoshell-mediated remote myotube activation. ACS Nano 11, 2494-2508. doi:10.1021/acsnano.6b08202

Marino, A., Genchi, G. G., Sinibaldi, E., and Ciofani, G. (2017b). Piezoelectric effects of materials on bio-interfaces. ACS Appl. Mater. Interfaces 9, 17663-17680. doi:10.1021/acsami.7b04323

Marino, A., Tonda-Turo, C., De Pasquale, D., Ruini, F., Genchi, G., Nitti, S., et al. (2017c). Gelatin/nanoceria nanocomposite fibers as antioxidant scaffolds for neuronal regeneration. Biochim. Biophys. Acta 1861, 386-395. doi:10.1016/j. bbagen.2016.11.022

Marino, A., Arai, S., Hou, Y., Sinibaldi, E., Pellegrino, M., Chang, Y. T., et al. (2015). Piezoelectric nanoparticle-assisted wireless neuronal stimulation. ACS Nano 9 , 7678-7689. doi:10.1021/acsnano.5b03162

Miyako, E., Russier, J., Mauro, M., Cebrian, C., Yawo, H., Menard-Moyon, C., et al. (2014). Photofunctional nanomodulators for bioexcitation. Angew. Chem. Int. Ed. Engl. 53, 13121-13125. doi:10.1002/anie.201407169

Mocan, L., Tabaran, F. A., Mocan, T., Bele, C., Orza, A. I., Lucan, C., et al. (2011). Selective ex-vivo photothermal ablation of human pancreatic cancer with albumin functionalized multiwalled carbon nanotubes. Int. J. Nanomedicine 6, 915-928. doi:10.2147/IJN.S19013

Mohanta, Y. K., Panda, S. K., Jayabalan, R., Sharma, N., Bastia, A. K., and Mohanta, T.K. (2017). Antimicrobial, antioxidant and cytotoxic activity of silver nanoparticles synthesized by leaf extract of Erythrina suberosa (Roxb.). Front. Mol. Biosci. 4:14. doi:10.3389/fmolb.2017.00014 
Mura, S., Nicolas, J., and Couvreur, P. (2013). Stimuli-responsive nanocarriers for drug delivery. Nat. Mater. 12, 991-1003. doi:10.1038/nmat3776

Nair, M., Guduru, R., Liang, P., Hong, J., Sagar, V., and Khizroev, S. (2013). Externally controlled on-demand release of anti-HIV drug using magneto-electric nanoparticles as carriers. Nat. Commun. 4, 1707. doi:10.1038/ ncomms 2717

Noh, S.-H., Moon, S. H., Shin, T.-H., Lim, Y., and Cheon, J. (2017). Recent advances of magneto-thermal capabilities of nanoparticles: from design principles to biomedical applications. Nano Today 13, 61-76. doi:10.1016/j. nantod.2017.02.006

Paviolo, C., and Stoddart, P. R. (2017). Gold nanoparticles for modulating neuronal behavior. Nanomaterials (Basel) 7, E92. doi:10.3390/nano7040092

Paz, J. T., Davidson, T. J., Frechette, E. S., Delord, B., Parada, I., Peng, K., et al. (2013). Closed-loop optogenetic control of thalamus as a tool for interrupting seizures after cortical injury. Nat. Neurosci. 16, 64-70. doi:10.1038/ nn.3269

Rau, L. R., Huang, W. Y., Liaw, J. W., and Tsai, S. W. (2016). Photothermal effects of laser-activated surface plasmonic gold nanoparticles on the apoptosis and osteogenesis of osteoblast-like cells. Int. J. Nanomedicine 11, 3461-3473. doi:10.2147/IJN.S108152

Robinson, J. T., Tabakman, S. M., Liang, Y., Wang, H., Casalongue, H. S., Vinh, D., et al. (2011). Ultrasmall reduced graphene oxide with high near-infrared absorbance for photothermal therapy. J. Am. Chem. Soc. 133, 6825-6831. doi:10.1021/ja2010175

Rocca, A., Mattoli, V., Mazzolai, B., and Ciofani, G. (2014). Cerium oxide nanoparticles inhibit adipogenesis in rat mesenchymal stem cells: potential therapeutic implications. Pharm. Res. 31, 2952-2962. doi:10.1007/s11095014-1390-7

Rocca, A., Moscato, S., Ronca, F., Nitti, S., Mattoli, V., Giorgi, M., et al. (2015). Pilot in vivo investigation of cerium oxide nanoparticles as a novel anti-obesity pharmaceutical formulation. Nanomedicine 11, 1725-1734. doi:10.1016/j. nano.2015.05.001

Rodzinski, A., Guduru, R., Liang, P., Hadjikhani, A., Stewart, T., Stimphil, E., et al. (2016). Targeted and controlled anticancer drug delivery and release with magnetoelectric nanoparticles. Sci. Rep. 6, 20867. doi:10.1038/srep20867

Royo-Gascon, N., Wininger, M., Scheinbeim, J. I., Firestein, B. L., and Craelius, W. (2013). Piezoelectric substrates promote neurite growth in rat spinal cord neurons. Ann. Biomed. Eng. 41, 112-122. doi:10.1007/s10439-012-0628-y

Schlatholter, T., Eustache, P., Porcel, E., Salado, D., Stefancikova, L., Tillement, O., et al. (2016). Improving proton therapy by metal-containing nanoparticles: nanoscale insights. Int. J. Nanomedicine 11, 1549-1556. doi:10.2147/IJN.S99410

Shapiro, M. G., Homma, K., Villarreal, S., Richter, C. P., and Bezanilla, F. (2012). Infrared light excites cells by changing their electrical capacitance. Nat. Commun. 3, 736. doi:10.1038/ncomms 1742

Srinivasan, M., Rajabi, M., and Mousa, S. A. (2015). Multifunctional nanomaterials and their applications in drug delivery and cancer therapy. Nanomaterials (Basel) 5, 1690-1703. doi:10.3390/nano5041690

Stanley, S. A., Gagner, J. E., Damanpour, S., Yoshida, M., Dordick, J. S., and Friedman, J. M. (2012). Radio-wave heating of iron oxide nanoparticles can regulate plasma glucose in mice. Science 336, 604-608. doi:10.1126/science. 1216753

Stanley, S. A., Sauer, J., Kane, R. S., Dordick, J. S., and Friedman, J. M. (2015). Remote regulation of glucose homeostasis in mice using genetically encoded nanoparticles. Nat. Med. 21, 92-98. doi:10.1038/nm.3730

Subiel, A., Ashmore, R., and Schettino, G. (2016). Standards and methodologies for characterizing radiobiological impact of high- $\mathrm{Z}$ nanoparticles. Theranostics 6 , 1651-1671. doi:10.7150/thno.15019

Tapeinos, C., Efthimiadou, E. K., Boukos, N., Charitidis, C. A., Koklioti, M., and Kordas, G. (2013). Microspheres as therapeutic delivery agents: synthesis and biological evaluation of $\mathrm{pH}$ responsiveness. J. Mater. Chem. B 1, 194-203. doi:10.1039/c2tb00013j

Tapeinos, C., Efthimiadou, E. K., Boukos, N., and Kordas, G. (2016). Sustained release profile of quatro stimuli nanocontainers as a multi sensitive vehicle exploiting cancer characteristics. Colloids Surf. B. Biointerfaces 148, 95-103. doi:10.1016/j.colsurfb.2016.08.019

Tapeinos, C., Kartsonakis, I., Liatsi, P., Daniilidis, I., and Kordas, G. (2008). Synthesis and characterization of magnetic nanocontainers. J. Am. Ceram. Soc. 91, 1052-1056. doi:10.1111/j.1551-2916.2007.02240.x

Tapeinos, C., Larrañaga, A., Sarasua, J.-R., Pandit, A., Larranaga, A., Sarasua, J.-R., et al. (2017). Functionalised collagen spheres reduce $\mathrm{H}_{2} \mathrm{O}_{2}$ mediated apoptosis by scavenging overexpressed ROS. Nanomed. Nanotechnol. Biol. Med. doi:10.1016/j.nano.2017.03.022

Tapeinos, C., and Pandit, A. (2016). Physical, chemical, and biological structures based on ROS-sensitive moieties that are able to respond to oxidative microenvironments. Adv. Mater. Weinheim 28, 5553-5585. doi:10.1002/adma. 201505376

Theato, P., Sumerlin, B. S., O’Reilly, R. K., and Epps, T. H. III (2013). Stimuli responsive materials. Chem. Soc. Rev. 42, 7055-7056. doi:10.1039/c3cs90057f

Torchilin, V. P. (2014). Multifunctional, stimuli-sensitive nanoparticulate systems for drug delivery. Nat. Rev. Drug Discov. 13, 813-827. doi:10.1038/nrd4333

Tortiglione, C., Antognazza, M. R., Tino, A., Bossio, C., Marchesano, V., Bauduin, A., et al. (2017). Semiconducting polymers are light nanotransducers in eyeless animals. Sci. Adv. 3, e1601699. doi:10.1126/sciadv.1601699

Tufail, Y., Yoshihiro, A., Pati, S., Li, M. M., and Tyler, W. J. (2011). Ultrasonic neuromodulation by brain stimulation with transcranial ultrasound. Nat. Protoc. 6, 1453-1470. doi:10.1038/nprot.2011.371

Wang, S., Riedinger, A., Li, H., Fu, C., Liu, H., Li, L., et al. (2015). Plasmonic copper sulfide nanocrystals exhibiting near-infrared photothermal and photodynamic therapeutic effects. ACS Nano 9, 1788-1800. doi:10.1021/ nn506687t

Wang, X., Song, J., Liu, J., and Wang, Z. L. (2007). Direct-current nanogenerator driven by ultrasonic waves. Science 316, 102-105. doi:10.1126/science.1139366

Wang, Y., and Guo, L. (2016). Nanomaterial-enabled neural stimulation. Front. Neurosci. 10:69. doi:10.3389/fnins.2016.00069

Xu, C., Lin, Y., Wang, J., Wu, L., Wei, W., Ren, J., et al. (2013). Nanoceria-triggered synergetic drug release based on $\mathrm{CeO}(2)$-capped mesoporous silica host-guest interactions and switchable enzymatic activity and cellular effects of $\mathrm{CeO}(2)$. Adv. Healthc. Mater. 2, 1591-1599. doi:10.1002/adhm.201200464

Yong, J., Needham, K., Brown, W. G., Nayagam, B. A., McArthur, S. L., Yu, A., et al. (2014). Gold-nanorod-assisted near-infrared stimulation of primary auditory neurons. Adv. Healthc. Mater. 3, 1862-1868. doi:10.1002/adhm.201400027

Yoo, S., Hong, S., Choi, Y., Park, J. H., and Nam, Y. (2014). Photothermal inhibition of neural activity with near-infrared-sensitive nanotransducers. ACS Nano 8, 8040-8049. doi:10.1021/nn5020775

Yue, K., Guduru, R., Hong, J., Liang, P., Nair, M., and Khizroev, S. (2012). Magnetoelectric nano-particles for non-invasive brain stimulation. PLoS ONE 7:e44040. doi:10.1371/journal.pone.0044040

Conflict of Interest Statement: The authors declare that the research was conducted in the absence of any commercial or financial relationships that could be construed as a potential conflict of interest.

Copyright $\odot 2017$ Genchi, Marino, Tapeinos and Ciofani. This is an open-access article distributed under the terms of the Creative Commons Attribution License (CC BY). The use, distribution or reproduction in other forums is permitted, provided the original author(s) or licensor are credited and that the original publication in this journal is cited, in accordance with accepted academic practice. No use, distribution or reproduction is permitted which does not comply with these terms. 